\title{
Dependence of eigenvalues of $2 n$th order boundary value transmission problems
}

\author{
Kun Li ${ }^{*}$, Jiong Sun and Xiaoling Hao
}

"Correspondence: qslikun@163.com Department of Mathematics, Inner Mongolia University, Hohhot, 010021, China

\begin{abstract}
The present paper deals with the dependence of eigenvalues of 2 nth order boundary value transmission problems on the problem. The eigenvalues depend not only continuously but also smoothly on the problem. Some new differential expressions of eigenvalues with respect to an endpoint, a coefficient, the weight function, boundary conditions, and transmission conditions, are given.
\end{abstract}

MSC: 34B24; 34L15

Keywords: transmission conditions; dependence of eigenvalues; continuity; differential expression

\section{Introduction}

It is well known that boundary value transmission problems are of great importance for their wide applications in physics and engineering. These problems, such as heat, mass transfer (see [1]), and diffraction problems, relate to discontinuous material properties, and their miscellaneous physical applications connected with these problems are found in many literature works, see, e.g., [1-17] and the corresponding references cited therein. To deal with interior discontinuities, some conditions are imposed on the discontinuous points, which are often called transmission conditions (see $[2,3,7,10,18,19])$, interface conditions (see [14, 17]), or point interactions (see [5]).

Eigenvalue problems play an important role in the theory of differential operators. There are several methods to characterize the eigenvalues of boundary value problems (see, for example, [20-22]), in particular, on the existence of solutions for linear or nonlinear Sturm-Liouville problems and higher order boundary value problems, we refer to [22-24]. In the classical case, i.e., without discontinuous points, Dauge and Helffer in [25] found that the Neumann eigenvalues are differentiable functions of the right endpoint $b$ satisfying a differential equation of the form

$$
\lambda^{\prime}=u^{2}(q-\lambda w)
$$

They also obtained the differential expression for the Dirichlet eigenvalues

$$
\lambda^{\prime}=-p u^{\prime 2},
$$

(c) The Author(s) 2017. This article is distributed under the terms of the Creative Commons Attribution 4.0 International License (http://creativecommons.org/licenses/by/4.0/), which permits unrestricted use, distribution, and reproduction in any medium, provided you give appropriate credit to the original author(s) and the source, provide a link to the Creative Commons license, and indicate if changes were made. 
which is sometimes called Hadamard's formula. In [26], Kong and Zettl gave a different proof of the Dauge-Helffer theorem and obtained a similar result for coupled boundary conditions in the case where the space $L^{2}(a, b)$ is replaced by $L^{1}(a, b)$. In [27], Kong and Zettl showed the continuity and differentiability of eigenvalues for regular Sturm-Liouville problems with respect to all the parameters and obtained differential expressions of the eigenvalues. Corresponding results for fourth order case were also obtained by Suo and Wang in [28]. Kong et al. generalized the continuity and differentiability of eigenvalues to higher order case in [24]. The obtained results on the properties of eigenvalues and eigenfunctions play an important role in the Bailey, Everitt, and Zettl code SLEIGN2 (see [29]). The major general purpose code for the numerical computation of the eigenvalues and eigenfunctions of boundary value problems is SLEUTH (see [30]). In recent papers, Zhang and Wang in [14] considered the discontinuous Sturm-Liouville eigenvalue problems, and obtained the differential expressions of eigenvalues with respect to the data. Zhang et al. also studied singular eigenvalue problems in [15]. Li et al. considered the fourth order discontinuous case and obtained some new differential expressions of the eigenvalues in [19].

In this paper, we study the dependence of eigenvalues of $2 n$th order boundary value transmission problems on the problem. Using the ideas of Mukhtarov and Yakubov [9] and Wang et al. [10], a new Hilbert space is constructed, in which the considered problems are put. We prove that if $\lambda$ is an eigenvalue of the considered problem, then $\lambda$ can be embedded in a continuous eigenvalue branch. We also give some new differential expressions of the eigenvalues, which generalize the previous results obtained by Kong et al. (see [24]).

This paper is composed as follows. We give some notations and preliminaries in Section 2. The continuity results of eigenvalues and eigenfunctions are obtained in Section 3. Section 4 presents differential expressions of the eigenvalues with respect to all the data.

\section{Notations and preliminaries}

Consider the $2 n$th order symmetric differential equation

$$
\begin{aligned}
& M_{P}(y)=\frac{1}{w} \sum_{k=0}^{n}(-1)^{k}\left(p_{n-k}(x) y^{(k)}\right)^{(k)}=\lambda y, \\
& \text { on } \quad J^{\prime}=\left(a^{\prime}, c\right) \cup\left(c, b^{\prime}\right), \quad-\infty \leq a^{\prime}<c<b^{\prime} \leq+\infty .
\end{aligned}
$$

Let

$$
J=J_{1} \cup J_{2}, \quad J_{1}=[a, c), J_{2}=(c, b], a^{\prime}<a<c<b<b^{\prime} .
$$

Consider the boundary conditions

$$
A Y(a)+B Y(b)=0
$$

and transmission conditions

$$
Y(c-)=C Y(c+),
$$


where

$$
p_{0}^{-1}(x), p_{1}(x), \ldots, p_{n}(x), \quad w(x) \in L_{\mathrm{loc}}\left(J^{\prime}, \mathbb{R}\right), \quad w(x)>0 \text { a.e. on } J^{\prime}
$$

Here $\lambda$ is the spectral parameter, $A=\left(a_{i j}\right)$ and $B=\left(b_{i j}\right)$ are $2 n \times 2 n$ complex matrices, $C=\left(c_{i j}\right)$ is $2 n \times 2 n$ real matrix, $\operatorname{det} C=\rho^{n}, \rho>0$ and satisfy

$$
\begin{aligned}
& \operatorname{rank}(A \mid B)=2 n, \\
& \rho A Q_{2 n} A^{*}=B Q_{2 n} B^{*}, \quad C^{*} Q_{2 n} C=\rho Q_{2 n},
\end{aligned}
$$

where

$$
\begin{aligned}
& Q_{2 n}=\left(\begin{array}{cc}
0 & E_{n} \\
-E_{n} & 0
\end{array}\right), \\
& E_{n}=\left(\begin{array}{llll} 
& & & 1 \\
& & 1 & \\
& . & & \\
1 & & &
\end{array}\right) \text {, } \\
& Y(x)=\left(\begin{array}{c}
y(x) \\
y^{[1]}(x) \\
\vdots \\
y^{[2 n-2]}(x) \\
y^{[2 n-1]}(x)
\end{array}\right) .
\end{aligned}
$$

Here $y^{[1]}(x), y^{[2]}(x), \ldots, y^{[2 n-1]}(x)$ are called quasi-derivatives of $y$ which are defined by (see [31])

$$
\begin{aligned}
& y^{[k]}(x)=\frac{d^{k} y}{d x^{k}}, \quad k=0,1, \ldots, n-1, \\
& y^{[n]}(x)=p_{0} \frac{d^{n} y}{d x^{n}}, \\
& y^{[n+k]}(x)=p_{k} \frac{d^{n-k} y}{d x^{n-k}}-\frac{d}{d x} y^{[n+k-1]}, \quad k=1,2, \ldots, n .
\end{aligned}
$$

Let $R_{z}(x)=\left(z^{[1]}(x), z^{[2]}(x), \ldots, z^{[2 n-1]}(x)\right), C_{y}(x)=\left(y^{[1]}(x), y^{[2]}(x), \ldots, y^{[2 n-1]}(x)\right)^{T}$, the equation

$$
M_{P}(y) \bar{z}-y M_{P}(\bar{z})=\frac{d}{d x}[y, z](x)
$$

is called Lagrange formula [31], where

$$
\begin{aligned}
{[y, z](x) } & =W(y, \bar{z} ; x) \\
& =\sum_{k=1}^{n}\left\{y^{[k-1]}(x) \bar{z}^{[2 n-k]}(x)-y^{[2 n-k]}(x) \bar{z}^{[k-1](x)}\right\} \\
& =R_{y}(x) Q_{2 n} C_{\bar{z}}(x) .
\end{aligned}
$$


Following Mukhtarov and Yakubov [9] and Wang et al. [10], we construct a new inner product in the Hilbert space $H=L_{w}^{2}\left(J_{1}\right) \oplus L_{w}^{2}\left(J_{2}\right)$ and a self-adjoint operator defined on $H$ such that the eigenvalues of (2.1)-(2.3) coincide with the spectra of this operator. To this end, the inner product is defined by

$$
\langle f, g\rangle=\int_{a}^{c} f \bar{g} w d x+\rho \int_{c}^{b} f \bar{g} w d x
$$

for all $f, g \in H$.

Let us consider the operator $L$ with domain

$$
\begin{aligned}
& D(L)=\left\{f \in H \mid f, f^{[1]}, \ldots, f^{[2 n-1]} \in A C_{\mathrm{loc}}(J),\right. \\
& \quad A F(a)+B F(b)=0, F(c-)=C F(c+), L f \in H\}, \\
& L f=M_{P}(f), \quad f \in D(L) .
\end{aligned}
$$

Lemma 1 (See [12]) Let (2.4)-(2.6) hold, the operator $L$ be defined as in (2.9). Then $L$ is a self-adjoint operator in $H$. The eigenvalues of $L$ are real, and they are finite or countably infinite without finite accumulation point.

\section{Continuity of eigenvalues and eigenfunctions}

In this section, we prove the continuity of eigenvalues and normalized eigenfunctions for the $2 n$th order boundary value transmission problems. Moreover, the characterization of the eigenvalues as zeros of an entire function is established.

Denoted by $\varphi_{11}(x, \lambda), \varphi_{12}(x, \lambda), \ldots, \varphi_{1 n}(x, \lambda)$ and $\chi_{11}(x, \lambda), \chi_{12}(x, \lambda), \ldots, \chi_{1 n}(x, \lambda)$ the solutions of (2.1) on the interval $[a, c)$ satisfy the initial conditions

$$
\left(C_{\varphi_{11}}, C_{\varphi_{12}}, \ldots, C_{\varphi_{1 n}}, C_{\chi_{11}}, C_{\chi_{12}}, \ldots, C_{\chi_{1 n}}\right)(a, \lambda)=I
$$

where $I$ is the identity matrix. Obviously, the above solutions are linearly independent.

Let $\varphi_{21}(x, \lambda), \varphi_{22}(x, \lambda), \ldots, \varphi_{2 n}(x, \lambda)$ and $\chi_{21}(x, \lambda), \chi_{22}(x, \lambda), \ldots, \chi_{2 n}(x, \lambda)$ be the solutions of equation (2.1) on the interval $(c, b]$ satisfying the conditions

$$
\begin{aligned}
& \left(C_{\varphi_{11}}, C_{\varphi_{12}}, \ldots, C_{\varphi_{1 n}}, C_{\chi_{11}}, C_{\chi_{12}}, \ldots, C_{\chi_{1 n}}\right)(c-, \lambda) \\
& \quad=C \cdot\left(C_{\varphi_{21}}, C_{\varphi_{22}}, \ldots, C_{\varphi_{2 n}}, C_{\chi_{21}}, C_{\chi_{22}}, \ldots, C_{\chi_{2 n}}\right)(c+, \lambda) .
\end{aligned}
$$

According to the properties of dependence of the solutions on the parameter, the Wronskian

$$
w_{i}(\lambda)=W\left(\varphi_{i 1}(x, \lambda), \varphi_{i 2}(x, \lambda), \ldots, \varphi_{i n}(x, \lambda), \chi_{i 1}(x, \lambda), \chi_{i 2}(x, \lambda), \ldots, \chi_{i n}(x, \lambda)\right) \quad(i=1,2)
$$

are independent of the variable $x$ and are entire functions of parameter $\lambda$. Short calculation yields that

$$
w_{2}(\lambda)=\frac{1}{\rho^{n}} w_{1}(\lambda),
$$


which implies that $\varphi_{21}(x, \lambda), \varphi_{22}(x, \lambda), \ldots, \varphi_{2 n}(x, \lambda)$ and $\chi_{21}(x, \lambda), \chi_{22}(x, \lambda), \ldots, \chi_{2 n}(x, \lambda)$ are linearly independent on the interval $(c, b]$.

Let

$$
\Phi_{1}(x, \lambda)=\left(C_{\varphi_{11}}, C_{\varphi_{12}}, \ldots, C_{\varphi_{1 n}}, C_{\chi_{11}}, C_{\chi_{12}}, \ldots, C_{\chi_{1 n}}\right)(x, \lambda), \quad x \in[a, c)
$$

and

$$
\Phi_{2}(x, \lambda)=\left(C_{\varphi_{21}}, C_{\varphi_{22}}, \ldots, C_{\varphi_{2 n}}, C_{\chi_{21}}, C_{\chi_{22}}, \ldots, C_{\chi_{2 n}}\right)(x, \lambda), \quad x \in(c, b], \lambda \in \mathbb{C}
$$

where $\Phi_{1}(c, \lambda)$ and $\Phi_{2}(c, \lambda)$ are defined by left and right limits. Let

$$
\Phi(x, \lambda)= \begin{cases}\Phi_{1}(x, \lambda), & x \in[a, c), \\ \Phi_{2}(x, \lambda), & x \in(c, b]\end{cases}
$$

and $\Phi(c-, \lambda)=\Phi_{1}(c, \lambda), \Phi(c+, \lambda)=\Phi_{2}(c, \lambda)$. For arbitrary $x \in J, \Phi(x, \lambda)$ is an entire function of $\lambda$.

Lemma 2 A complex number $\lambda_{0}$ is an eigenvalue of the operator $L$ if and only if

$$
\Delta\left(\lambda_{0}\right)=\operatorname{det}\left(A+B \Phi\left(b, \lambda_{0}\right)\right)=0
$$

Proof Let $\lambda_{0}$ be an eigenvalue of $L$ and $u_{0}(x)$ be the corresponding eigenfunction. Then $u_{0}(x)$ can be represented by (see [12])

$$
u_{0}(x)=\left\{\begin{array}{l}
c_{1} \varphi_{11}\left(x, \lambda_{0}\right)+\cdots+c_{n} \varphi_{1 n}\left(x, \lambda_{0}\right)+c_{n+1} \chi_{11}\left(x, \lambda_{0}\right)+\cdots+c_{2 n} \chi_{1 n}\left(x, \lambda_{0}\right), \\
x \in[a, c), \\
c_{1} \varphi_{21}\left(x, \lambda_{0}\right)+\cdots+c_{n} \varphi_{2 n}\left(x, \lambda_{0}\right)+c_{n+1} \chi_{21}\left(x, \lambda_{0}\right)+c_{2 n} \chi_{2 n}\left(x, \lambda_{0}\right), \\
x \in(c, b]
\end{array}\right.
$$

where at least one of coefficients $c_{i}(i=1,2, \ldots, 2 n)$ is not zero. Substituting $u_{0}(x)$ into boundary conditions (2.2) yields

$$
\begin{aligned}
& A\left(C_{\varphi_{11}}, \ldots, C_{\varphi_{1 n}}, C_{\chi_{11}}, \ldots, C_{\chi_{1 n}}\right)(a, \lambda)\left(c_{1}, \ldots, c_{2 n}\right)^{T} \\
& \quad+B\left(C_{\varphi_{21}}, \ldots, C_{\varphi_{2 n}}, C_{\chi_{21}}, \ldots, C_{\chi_{2 n}}\right)(b, \lambda)\left(c_{1}, \ldots, c_{2 n}\right)^{T}=0 .
\end{aligned}
$$

By (3.1), (3.4), and (3.5), one gets that

$$
\left(A+B \Phi\left(b, \lambda_{0}\right)\right)\left(c_{1}, \ldots, c_{2 n}\right)^{T}=0 .
$$

Since $c_{1}, \ldots, c_{2 n}$ are not all zero, $\operatorname{det}\left(A+B \Phi\left(b, \lambda_{0}\right)\right)=0$. 
On the contrary, if $\operatorname{det}\left(A+B \Phi\left(b, \lambda_{0}\right)\right)=0$, then the homogeneous system of the linear equations (3.6) for the constants $c_{i}(i=1, \ldots, 2 n)$ has non-zero solution $\left(c_{1}^{\prime}, \ldots, c_{2 n}^{\prime}\right)$. Let

$$
u(x)=\left\{\begin{array}{l}
c_{1}^{\prime} \varphi_{11}\left(x, \lambda_{0}\right)+\cdots+c_{n}^{\prime} \varphi_{1 n}\left(x, \lambda_{0}\right)+c_{n+1}^{\prime} \chi_{11}\left(x, \lambda_{0}\right)+\cdots+c_{2 n}^{\prime} \chi_{1 n}\left(x, \lambda_{0}\right), \\
\quad x \in[a, c), \\
c_{1}^{\prime} \varphi_{21}\left(x, \lambda_{0}\right)+\cdots+c_{n}^{\prime} \varphi_{2 n}\left(x, \lambda_{0}\right)+c_{n+1}^{\prime} \chi_{21}\left(x, \lambda_{0}\right)+\cdots+c_{2 n}^{\prime} \chi_{2 n}\left(x, \lambda_{0}\right), \\
x \in(c, b],
\end{array}\right.
$$

then $u(x)$ is the non-trivial solution of equation $L u=\lambda u$ satisfying conditions (2.2) and (2.3). Therefore, $\lambda_{0}$ is an eigenvalue of $L$.

In the following, we introduce the notation

$$
\Omega=\left\{\omega=\left(a, b, A, B, C, 1 / p_{0}, p_{1}, \ldots, p_{n}, w\right)\right\}
$$

such that (2.2)-(2.6) hold.

We aim to illustrate the continuous dependence of eigenvalues and eigenfunctions on the problem, i.e., one small change of the problem only results in a diminutive change of each eigenvalue and eigenfunction. This means we need to compare the spectra of different problems which may be defined on different intervals determined by different $\omega$. From the definition of $\Omega$, we know that each of $\omega \in \Omega$ uniquely determines a boundary value transmission problem. And the values of $\frac{1}{p_{0}}, p_{1}, \ldots, p_{n}, w$ outside the interval $J$, i.e., in $J^{\prime} \backslash J$, do not affect the spectrum determined by $\omega$. For these reasons, let

$$
\widetilde{\Omega}=\left\{\omega=\left(a, b, A, B, C, \widetilde{1 / p_{0}}, \tilde{p}_{1}, \ldots, \tilde{p}_{n}, \tilde{w}\right)\right\},
$$

where

$$
\widetilde{1 / p_{0}}= \begin{cases}1 / p_{0}, & x \in J \\ 0, & x \in J^{\prime} \backslash J\end{cases}
$$

and $\tilde{p}_{1}, \ldots, \tilde{p}_{n}, \tilde{w}$ have similar definitions. Then we investigate the Banach space which is defined as

$$
X=\mathbb{R} \times \mathbb{R} \times M_{2 n \times 2 n}(\mathbb{C}) \times M_{2 n \times 2 n}(\mathbb{C}) \times M_{2 n \times 2 n}(\mathbb{R}) \times \underbrace{L_{\left(a^{\prime}, b^{\prime}\right)} \times \cdots \times L_{\left(a^{\prime}, b^{\prime}\right)}}_{n+2},
$$

and its norm is given by

$$
\|\omega\|=|a|+|b|+\|A\|+\|B\|+\|C\|+\int_{a^{\prime}}^{b^{\prime}}\left(\left|\widetilde{1 / p_{0}}\right|+\sum_{i=1}^{n}\left|\tilde{p}_{i}\right|+|\tilde{w}|\right),
$$

where $\|\cdot\|$ is any fixed matrix norm. Because $1 / p_{0}, p_{1}, \ldots, p_{n}, w$ are only defined in $L_{\text {loc }}^{1}\left(J^{\prime}\right)$, $\Omega$ is not a subset of $X$, but $\widetilde{\Omega}$ is. To study the continuity of eigenvalues and eigenfunctions on the problem, $\Omega$ is assumed to be a subset of $X$ and inherits its norm from $X$ on which the convergence in $\Omega$ depends. Because every point in $\Omega$ is an accumulation point 
of $\Omega$ in relation to the norm (3.7), so it is meaningful to discuss convergence of boundary value transmission problems with respect to this norm. Based on the space $X$, the set $\Omega$ and Lemma 2 , we obtain that the eigenvalues of $2 n$th order boundary value transmission problems depend continuously on the problem.

Theorem 1 Let $\omega_{0}=\left(a_{0}, b_{0}, A_{0}, B_{0}, C_{0}, \frac{1}{p_{0_{0}}}, p_{1_{0}}, \ldots, p_{n_{0}}, w_{0}\right) \in \Omega$. Assume that $\mu=\lambda\left(\omega_{0}\right)$ is an eigenvalue of the operator $L$ determined by $\omega_{0}$. Then $\lambda$ is continuous at $\omega_{0}$, that is, given any $\varepsilon>0$, there exists $\delta>0$ such that

$$
\left\|\omega-\omega_{0}\right\|<\delta
$$

for any $\omega \in \Omega$, then

$$
\left|\lambda(\omega)-\lambda\left(\omega_{0}\right)\right|<\varepsilon
$$

Proof From Lemma 2, we get that for $\omega \in \Omega, \lambda(\omega)$ is an eigenvalue of the operator $L$ if and only if $\Delta(\omega, \lambda)=0$. For any $\omega \in \Omega, \Delta(\omega, \lambda)$ is an entire function of $\lambda$ and is continuous in $\omega$ (see [32], Theorems 2.7, 2.8), and $\Delta\left(\omega_{0}, \mu\right)=0$. Since the operator $L$ is self-adjoint, we know that $\mu$ is an isolated eigenvalue, and then $\Delta\left(\omega_{0}, \lambda\right)$ is not constant in $\lambda$. Hence there exists $\rho_{0}>0$ such that $\Delta\left(\omega_{0}, \lambda\right) \neq 0$ for $\lambda \in S_{\rho_{0}}:=\left\{\lambda \in \mathbb{C}:|\lambda-\mu|=\rho_{0}\right\}$. By the well known theorem on continuity of the roots of an equation as a function of parameters (see [33], 9.17.4), the proof for Theorem 1 is completed.

In what follows we will always assume that each eigenvalue $\lambda(\omega)$ is embedded in a continuous eigenvalue branch.

Lemma 3 Consider the initial value problem

$$
\left\{\begin{array}{l}
\sum_{k=0}^{n}(-1)^{k}\left(p_{n-k}(x) y^{(k)}\right)^{(k)}=\lambda w y, \\
y\left(t_{0}\right)=d_{0}, \quad y^{[1]}\left(t_{0}\right)=d_{1}, \quad \ldots, \quad y^{[2 n-1]}\left(t_{0}\right)=d_{2 n-1},
\end{array}\right.
$$

where $t_{0} \in[a, c) \cup(c, b] \cup\{c+, c-\}$. Then the unique solution

$$
y=\left(\cdot, t_{0}, d_{0}, \ldots, d_{2 n-1}, C, 1 / p_{0}, p_{1}, \ldots, p_{n}, w\right)
$$

satisfying the above mentioned initial conditions and transmission conditions (2.3) is a continuous function of all its variables. That is, for any $\varepsilon>0$, there exists $\delta>0$ such that if

$$
\left|t-t_{0}\right|+\sum_{i=0}^{2 n-1}\left|d_{i}-d_{i_{0}}\right|+\left\|C-C_{0}\right\|+\int_{a}^{b}\left(\left|\frac{1}{p_{0}}-\frac{1}{p_{0_{0}}}\right|+\sum_{i=1}^{n}\left|p_{i}-p_{i_{0}}\right|+\left|w-w_{0}\right|\right)<\delta,
$$

then

$$
\begin{aligned}
& \mid y\left(x, t, d_{0}, \ldots, d_{2 n-1}, C, \frac{1}{p_{0}}, p_{1}, \ldots, p_{n}, w\right) \\
& \quad-y\left(x, t_{0}, d_{0_{0}}, \ldots, d_{(2 n-1)}, C_{0}, \frac{1}{p_{0_{0}}}, p_{1_{0}}, \ldots, p_{n_{0}}, w_{0}\right) \mid<\varepsilon,
\end{aligned}
$$




$$
\begin{aligned}
& \quad \mid y^{[1]}\left(x, t, d_{0}, \ldots, d_{2 n-1}, C, \frac{1}{p_{0}}, p_{1}, \ldots, p_{n}, w\right) \\
& \quad-y^{[1]}\left(x, t_{0}, d_{0_{0}}, \ldots, d_{(2 n-1)_{0}}, C_{0}, \frac{1}{p_{0_{0}}}, p_{1_{0}}, \ldots, p_{n_{0}}, w_{0}\right) \mid<\varepsilon, \\
& \vdots \\
& \quad \mid y^{[2 n-1]}\left(x, t, d_{0}, \ldots, d_{2 n-1}, C, \frac{1}{p_{0}}, p_{1}, \ldots, p_{n}, w\right) \\
& \quad-y^{[2 n-1]}\left(x, t_{0}, d_{0_{0}}, \ldots, d_{(2 n-1)_{0}}, C_{0}, \frac{1}{p_{0_{0}}}, p_{1_{0}}, \ldots, p_{n_{0}}, w_{0}\right) \mid<\varepsilon
\end{aligned}
$$

uniformly for all $x \in J$.

Proof For $t_{0}=c-$ and $x=c+$, by transmission conditions (2.2) and $\operatorname{det} C=\operatorname{det} C_{0}=\rho^{n}>0$, the result holds for $x=c+$. By the extension of continuity of $y(x, \lambda)$ on $J_{1}$ or $J_{2}$, respectively, the statement can be seen from Lemma 3.1 in [27] when $x \in J$. As $t_{0}=c+$, utilizing the same method, the result follows. For $x \in J$, using Lemma 3.1 in [27] and the above method, the statement follows.

Lemma 4 Let $\omega_{0}=\left(a_{0}, b_{0}, A_{0}, B_{0}, C_{0}, \frac{1}{p_{0_{0}}}, p_{1_{0}}, \ldots, p_{n_{0}}, w_{0}\right)$. Let $\lambda=\lambda(\omega)$ be an eigenvalue of the operator $L$. If the multiplicity of $\lambda\left(\omega_{0}\right)$ is 1 , then there exists a neighborhood $N$ of $\omega_{0}$ belonging to $\Omega$ such that the multiplicity of $\lambda(\omega)$ is 1 for every $\omega$ in $N$.

Proof If $\lambda\left(\omega_{0}\right)$ is simple, then $\Delta^{\prime}\left(\lambda\left(\omega_{0}\right)\right) \neq 0$. Since $\Delta(\lambda)$ is an entire function of $\lambda$, then the conclusion follows from Theorem 1 .

A normalized eigenfunction $u$ of the operator $L$ means an eigenfunction $u$ satisfies

$$
\langle u, u\rangle=\int_{a}^{c} u \bar{u} w d x+\rho \int_{c}^{b} u \bar{u} w d x=1
$$

Theorem 2 Let the notation and hypotheses of Theorem 1 hold. If the multiplicity of eigenvalue $\lambda(\omega)$ is $l(l=1,2, \ldots, 2 n)$ for all $\omega \in N$, and $N \in \Omega$ is a neighborhood of $\omega_{0}$. Then there exist l linearly independent normalized eigenfunctions $u_{k}(\cdot, \omega)$ of $\lambda(\omega)$. As $\omega \rightarrow \omega_{0}$, we have

$$
\begin{aligned}
& u_{k}(\cdot, \omega) \rightarrow u_{k}\left(\cdot, \omega_{0}\right), \\
& u_{k}^{[j]}(\cdot, \omega) \rightarrow u_{k}^{[j]}\left(\cdot, \omega_{0}\right), \quad k=1,2, \ldots, l, j=1,2, \ldots, 2 n-1,
\end{aligned}
$$

uniformly on the interval J.

Particularly, if $\lambda\left(\omega_{0}\right)$ is simple for some $\omega_{0} \in \Omega$, then there exists a normalized eigenfunction $u_{1}=u_{1}(\cdot, \omega)$ such that $(3.8)$ holds for $k=1$.

Proof (a) If the multiplicity of $\lambda\left(\omega_{0}\right)$ is 1 , then, by Lemma 4 , there exists a neighborhood $N$ of $\omega_{0}$ such that the multiplicity of $\lambda(\omega)$ is 1 for any $\omega \in N$. For each $\omega \in N$, choose an eigenfunction $u=u(\cdot, \omega)$ of $\lambda(\omega)$ satisfying

$$
\left\|U\left(x_{0}, \omega\right)\right\|=\left|u\left(x_{0}, \omega\right)\right|+\left|u^{[1]}\left(x_{0}, \omega\right)\right|+\cdots+\left|u^{[2 n-1]}\left(x_{0}, \omega\right)\right|=1, \quad u(x, \omega)>0,
$$


for some $x_{0} \in(a, c) \cup(c, b)$ and $x$ near $x_{0}$, where $U(\cdot, \omega)=\left(u(\cdot, \omega), u^{[1]}(\cdot, \omega), \ldots, u^{[2 n-1]}(\cdot, \omega)\right)^{T}$. It is sufficient to prove that

$$
U\left(x_{0}, \omega\right) \rightarrow U\left(x_{0}, \omega_{0}\right), \quad \omega \rightarrow \omega_{0}, \omega \in \Omega
$$

If (3.9) is not fulfilled, then we can choose a sequence $\omega_{k} \rightarrow \omega_{0}$ such that

$$
U\left(x_{0}, \omega_{k}\right) \rightarrow Y, \quad \omega_{k} \rightarrow \omega_{0}, \omega \in \Omega
$$

Due to the normalization at $x_{0}, Y$ and $U\left(x_{0}, \omega_{0}\right)$ are two linearly independent vectors in $\mathbb{C}^{2 n}$. Let $Z(x)$ be the vector solution of (2.1) with $\omega=\omega_{0}, \lambda=\lambda\left(\omega_{0}\right)$ and the initial condition $Z\left(x_{0}\right)=Y$. By Lemma $3, U\left(x, \omega_{k}\right) \rightarrow Z(x)$ uniformly on $J$. Particularly,

$$
\begin{aligned}
& U\left(a, \omega_{k}\right) \rightarrow Z(a), \quad U\left(b, \omega_{k}\right) \rightarrow Z(b), \\
& U\left(c-, \omega_{k}\right) \rightarrow Z(c-), \quad U\left(c+, \omega_{k}\right) \rightarrow Z(c+) .
\end{aligned}
$$

Since $U\left(\cdot, \omega_{k}\right)$ satisfies the conditions

$$
A_{k} U\left(a, \omega_{k}\right)+B_{k} U\left(b, \omega_{k}\right)=0, \quad U\left(c-, \omega_{k}\right)=C_{k} U\left(c+, \omega_{k}\right),
$$

by taking limits as $k \rightarrow \infty$, we obtain that

$$
A_{0} Z(a)+B_{0} Z(b)=0, \quad Z(c-)=C_{0} Z(c+) .
$$

Hence $Z(x)$ is a vector eigenfunction for $\omega=\omega_{0}$ and $\lambda=\lambda\left(\omega_{0}\right)$, which contradicts the fact that $\lambda\left(\omega_{0}\right)$ is simple.

Again by Lemma $3, u(x, \omega) \rightarrow u\left(x, \omega_{0}\right), u^{[1]}(x, \omega) \rightarrow u^{[1]}\left(x, \omega_{0}\right), \ldots, u^{[2 n-1]}(x, \omega) \rightarrow$ $u^{[2 n-1]}\left(x, \omega_{0}\right)$ as $\omega \rightarrow \omega_{0}$, and $x \in J$. The conclusion follows.

(b) If the multiplicity of $\lambda(\omega)$ is $l(l=2, \ldots, 2 n)$ for all $\omega$ in some neighborhood $N$ of $\omega_{0}$ in $\Omega$. Then we can choose eigenfunctions of $\lambda(\omega)$ satisfying the same initial conditions at $c_{0}$ for some $c_{0} \in J$ since a linear combination of $l$ linearly independent eigenfunctions can be chosen to satisfy arbitrary initial conditions.

The above discussion illustrates that for every self-adjoint boundary value transmission problem and every eigenvalue $\lambda(\omega)$, the eigenfunction $u(\cdot, \omega)$ and its quasi-derivatives $u^{[1]}(\cdot, \omega), \ldots, u^{[2 n-1]}(\cdot, \omega)$ are uniformly convergent in $\omega$ for $x \in J$. Then we normalize the eigenfunctions to end the proof.

\section{Differential expressions of eigenvalues on the problem}

In this section, we will obtain the differential expressions of eigenvalues with respect to the data. To this end, we will use Frechet derivative and list its definition as follows.

Definition 1 (See [24]) Let $X, Y$ be Banach spaces. A map $T: X \rightarrow Y$ is Frechet differentiable at a given point $x \in X$ if a bounded linear operator $d T_{x}: X \rightarrow Y$ satisfies that for $h \in X$

$$
\left|T(x+h)-T(x)-d T_{x}(h)\right|=o(h) \quad \text { as } h \rightarrow 0 .
$$


Lemma 5 (See [24]) Assume a real-valued function $f \in L_{\mathrm{loc}}(a, b)$. Then

$$
\lim _{h \rightarrow 0} \frac{1}{h} \int_{x}^{x+h} f=f(x) \text { a.e. in }(a, b) .
$$

Lemma 6 Suppose that $P=\left(p_{0}, p_{1}, \ldots, p_{n}\right)$ and $Q=\left(q_{0}, q_{1}, \ldots, q_{n}\right)$. Then we have

$$
\begin{aligned}
& \left\langle M_{P} y, z\right\rangle-\left\langle y, M_{Q} z\right\rangle \\
& =[y, z]_{a}^{c}+\rho[y, z]_{c}^{b}+\int_{a}^{c} \sum_{k=0}^{n-1}(-1)^{n+k}\left(p_{n-k}-q_{n-k}\right) y^{(k)} \bar{z}^{(k)}+\int_{a}^{c}\left(p_{0}-q_{0}\right) y^{(n)} \bar{z}^{(n)} \\
& \quad+\rho \int_{c}^{b} \sum_{k=0}^{n-1}(-1)^{n+k}\left(p_{n-k}-q_{n-k}\right) y^{(k)} \bar{z}^{(k)}+\rho \int_{c}^{b}\left(p_{0}-q_{0}\right) y^{(n)} \bar{z}^{(n)} .
\end{aligned}
$$

Proof This follows directly from integration by parts.

Theorem 3 Let $\omega=(a, b, A, B, C, P, w) \in \Omega$ with $P=\left(\frac{1}{p_{0}}, p_{1}, \ldots, p_{n}\right)$ and $\lambda=\lambda(\omega)$ be an eigenvalue of operator $L$ connected with $\omega$, and let $u=u(\cdot, \omega)$ be the corresponding eigenfunction. Assume that $\lambda(\omega)$ has constant geometric multiplicity in some neighborhood $N \subset \Omega$ for all fixed components of $\omega$ except one component.

1. Let all components of $\omega$ except $p_{k}$ for some $(k=1,2, \ldots, n)$ be fixed. Consider $\lambda$ as a function of $p_{k} \in L(J)$. Then $\lambda$ is Frechet differentiable at $p_{k}$ and

$$
d \lambda_{p_{k}}(h)=\int_{a}^{c}(-1)^{n+k}\left|u^{(k)}\right|^{2} h+\rho \int_{c}^{b}(-1)^{n+k}\left|u^{(k)}\right|^{2} h, \quad h \in L(J) .
$$

2. Let all components of $\omega$ except $1 / p_{0}$ be fixed. Consider $\lambda$ as a function of $1 / p_{0} \in L(J)$.

Then $\lambda$ is Frechet differentiable at $1 / p_{0}$ and

$$
d \lambda_{1 / p_{0}}(h)=-\left(\int_{a}^{c}\left|p_{0} u^{(n)}\right|^{2} h+\rho \int_{c}^{b}\left|p_{0} u^{(n)}\right|^{2} h\right), \quad h \in L(J) .
$$

3. Let all components of $\omega$ except $w$ be fixed. Consider $\lambda$ as a function of $w \in L(J)$. Then $\lambda$ is Frechet differentiable at $w$ and

$$
d \lambda_{w}(h)=-\lambda\left(\int_{a}^{c}|u|^{2} h+\rho \int_{c}^{b}|u|^{2} h\right), \quad h \in L(J) .
$$

Proof We only give the proofs of (4.2) and (4.3) since (4.4) can be proved similarly. Let $u=u\left(\cdot, p_{k}\right)$ and $v=u\left(\cdot, p_{k}+h\right)$ such that $u^{(k)}\left(\cdot, p_{k}+h\right) \rightarrow u^{(k)}\left(\cdot, p_{k}\right)(k=1,2, \ldots, n)$ uniformly on $J$ as $h \rightarrow 0$. From (2.1) and (4.1) it is obtained that

$$
\begin{aligned}
& {\left[\lambda\left(p_{k}+h\right)-\lambda\left(p_{k}\right)\right]\langle u, v\rangle} \\
& \quad=-[u, v]_{a}^{c}-\rho[u, v]_{c}^{b}+\int_{a}^{c}(-1)^{n+k} u^{(k)} \bar{v}^{(k)} h+\rho \int_{c}^{b}(-1)^{n+k} u^{(k)} \bar{v}^{(k)} h .
\end{aligned}
$$

Condition (2.6) implies

$$
[u, v]_{a}^{c}+\rho[u, v]_{c}^{b}=0,
$$


then by Theorem 1 and Theorem 2 we have

$$
\left[\lambda\left(p_{k}+h\right)-\lambda\left(p_{k}\right)\right](1+o(1))=\int_{a}^{c}(-1)^{n+k}\left|u^{(k)}\right|^{2} h+\rho \int_{c}^{b}(-1)^{n+k}\left|u^{(k)}\right|^{2} h+o(h),
$$

and hence

$$
\begin{aligned}
\lambda\left(p_{k}+h\right)-\lambda\left(p_{k}\right) & =\left[\int_{a}^{c}(-1)^{n+k}\left|u^{(k)}\right|^{2} h+\rho \int_{c}^{b}(-1)^{n+k}\left|u^{(k)}\right|^{2} h+o(h)\right](1+o(1)) \\
& =(-1)^{n+k} \int_{a}^{c}\left|u^{(k)}\right|^{2} h+(-1)^{n+k} \rho \int_{c}^{b}\left|u^{(k)}\right|^{2} h+o(h) \quad \text { as } h \rightarrow 0 .
\end{aligned}
$$

Therefore, the proof for (4.2) is completed.

In the following, we prove formula (4.3). Let $u=u\left(\cdot, \frac{1}{p_{0}}\right)$ and $v=u\left(\cdot, \frac{1}{q_{0}}\right)$ with $\frac{1}{q_{0}}=\frac{1}{p_{0}}+h$, and when $h \rightarrow 0, u\left(\cdot, \frac{1}{q_{0}}\right) \rightarrow u\left(\cdot, \frac{1}{p_{0}}\right)$. Then

$$
p_{0}-q_{0}=p_{0} q_{0} h
$$

Using (2.1) and the integration by parts, we can obtain

$$
\begin{aligned}
{\left[\lambda\left(\frac{1}{p_{0}}\right)-\lambda\left(\frac{1}{q_{0}}\right)\right]\langle u, v\rangle=} & {[u v]_{a}^{c}+\rho[u v]_{c}^{b}+\int_{a}^{c}\left(p_{0}-q_{0}\right) u^{(n)} \bar{v}^{(n)} } \\
& +\rho \int_{c}^{b}\left(p_{0}-q_{0}\right) u^{(n)} \bar{v}^{(n)} .
\end{aligned}
$$

From condition (2.6) we get that

$$
\left[\lambda\left(\frac{1}{q_{0}}\right)-\lambda\left(\frac{1}{p_{0}}\right)\right]\langle u, v\rangle=-\int_{a}^{c} p_{0} u^{(n)} q_{0} \bar{\nu}^{(n)} h+\rho \int_{c}^{b} p_{0} u^{(n)} q_{0} \bar{v}^{(n)} h
$$

Taking limits as $h \rightarrow 0$, we complete the proof of (4.3).

Theorem 4 Assume that the assumptions in Theorem 3 hold. Let all components of $\omega$ except $b$ be fixed, and let $\lambda=\lambda(b)$ and $u=u(\cdot, b)$. Then $\lambda$ is differentiable at $b$ and

$$
\lambda^{\prime}(b)=\rho \sum_{k=1}^{n}\left(u^{[k-1]}(b)\left(\bar{u}^{[2 n-k]}\right)^{\prime}(b)-u^{[2 n-k]}(b)\left(\bar{u}^{[k-1]}\right)^{\prime}(b)\right), \quad \text { a.e. for } b \in\left(c, b^{\prime}\right) .
$$

Proof For small $h$, choose $u=u(\cdot, b)$ and $v=u(\cdot, b+h)$. By (2.1), (2.6), and Lemma 6, we get that

$$
\begin{aligned}
& {[\lambda(b+h)-\lambda(b)]\langle u, v\rangle} \\
& \quad=-[u v]_{a}^{c}-\rho[u v]_{c}^{b}=[u v](a)-\rho[u v](b) \\
& \quad=R_{u}(a) Q_{2 n} C_{\bar{\nu}}(a)-\rho R_{u}(b) Q_{2 n} C_{\bar{\nu}}(b) \\
& \quad=R_{u}(b, b)\left(A^{-1} B\right) Q_{2 n} A^{-1} B C_{\bar{u}}(b+h, b+h)-\rho R_{u}(b, b) Q_{2 n} C_{\bar{u}}(b, b+h) \\
& \quad=\rho R_{u}(b, b) Q_{2 n} C_{\bar{u}}(b+h, b+h)-\rho R_{u}(b, b) Q_{2 n} C_{\bar{u}}(b, b+h)
\end{aligned}
$$




$$
\begin{aligned}
& =\rho R_{u}(b, b) Q_{2 n} \int_{b}^{b+h} C_{\bar{u}}^{\prime}(s, b+h) d s \\
& =\rho R_{u}(b, b) Q_{2 n}\left[\int_{b}^{b+h} C_{\bar{u}}^{\prime}(s, b) d s+o(h)\right] .
\end{aligned}
$$

Therefore, from Lemma 5 we have

$$
\begin{aligned}
\lambda(b+h)-\lambda(b) & =\rho R_{u}(b, b) Q_{2 n}\left[\int_{b}^{b+h} C_{\bar{u}}^{\prime}(s, b) d s+o(h)\right] \\
& =\rho h R_{u}(b, b) Q_{2 n} C_{\bar{u}}^{\prime}(b, b)+o(h) \quad \text { a.e. in }\left(c, b^{\prime}\right) .
\end{aligned}
$$

Dividing both sides of the above equality by $h$ and letting $h \rightarrow 0$ yield that

$$
\lambda^{\prime}(b)=\rho R_{u}(b, b) Q_{2 n} C_{\bar{u}}^{\prime}(b, b)=\rho\left[u, u^{\prime}\right](b) .
$$

Then the result follows from (2.8).

Theorem 5 Assume that the assumptions in Theorem 3 hold.

1. Let all components of $\omega$ except $A$ be fixed. Denote the eigenvalue and the normalized eigenfunction with respect to $A$ by $\lambda=\lambda(A)$ and $u=u(\cdot, A)$, respectively. For all $E$ satisfying $\rho(A+E) Q_{2 n}(A+E)^{*}=B Q_{2 n} B^{*}$ in the neighborhood of $A, \lambda$ is differentiable at $A$ and

$$
d \lambda_{A}(E)=-R_{u}(a) Q_{2 n} A^{-1} H C_{\bar{u}}(a) .
$$

2. Let all components of $\omega$ except $B$ be fixed. Denote the eigenvalue and the normalized eigenfunction with respect to $B$ by $\lambda=\lambda(B)$ and $u=u(\cdot, B)$, respectively. For all $E$ satisfying $\rho A Q_{2 n} A^{*}=(B+E) Q_{2 n}(B+E)^{*}$ in the neighborhood of $B, \lambda$ is differentiable at $B$ and

$$
d \lambda_{B}(E)=\rho R_{u}(b) Q_{2 n} B^{-1} H C_{\bar{u}}(b) .
$$

3. Let all components of $\omega$ except $C$ be fixed. Denote the eigenvalue and the normalized eigenfunction with respect to $C$ by $\lambda=\lambda(C)$ and $u=u(\cdot, C)$, respectively. For all $E$ satisfying $\operatorname{det}(C+E)=\rho^{n}$ and $(C+E)^{*} Q_{2 n}(C+E)=\rho Q_{2 n}$ in the neighborhood of $C, \lambda$ is differentiable at $C$ and

$$
d \lambda_{C}(E)=-R_{u}(c+) C^{*} Q_{2 n} H C_{\bar{u}}(c+) .
$$

Proof For small $E$, choose $u=u(\cdot, A), v=u(\cdot, A+E)$ such that $u(\cdot, A+E) \rightarrow u(\cdot, A)$ as $E \rightarrow 0$, then by condition (2.6) we have

$$
\begin{aligned}
& {[\lambda(A+E)-\lambda(A)]\langle u, v\rangle} \\
& \quad=[u, v](a)-\rho[u, v](b) \\
& \quad=R_{u}(a) Q_{2 n} C_{\bar{v}}(a)-\rho R_{u}(b) Q_{2 n} C_{\bar{v}}(b) \\
& \quad=R_{u}(a) Q_{2 n} C_{\bar{v}}(a)-\rho R_{u}(a)\left(B A^{-1}\right) Q_{2 n} B^{-1}(A+E) C_{\bar{v}}(b) .
\end{aligned}
$$


By (2.6) one gets that

$$
A^{*-1} Q_{2 n} A^{-1}=\rho B^{*-1} Q_{2 n} B^{-1},
$$

and hence

$$
\left(B^{-1} A\right)^{*} Q_{2 n} B^{-1} A=\frac{1}{\rho} Q_{2 n} .
$$

Substituting (4.10), (4.11) into (4.9) and letting $E \rightarrow 0$, we get that

$$
\lambda(A+E)-\lambda(A)=-R_{u}(a) Q_{2 n} A^{-1} E C_{\bar{u}}(a)+o(E),
$$

this completes the proof of (4.6). The proof for (4.7) is similar to this proof, hence is omitted here.

Let $u=u(\cdot, C), v=u(\cdot, C+E)$ and assume that when $E \rightarrow 0, u(\cdot, C+E) \rightarrow u(\cdot, C)$. Using the same method mentioned above, we can get

$$
[\lambda(C+E)-\lambda(C)]\langle u, v\rangle=-[u v]_{a}^{c}-\rho[u v]_{c}^{b} .
$$

By condition (2.6) and transmission conditions (2.3) we have

$$
\begin{aligned}
& {[\lambda(C+E)-\lambda(C)]\langle u, v\rangle} \\
& \quad=-[u v](c-)+\rho[u v](c+) \\
& \quad=\rho R_{u}(c+) Q_{2 n} C_{\bar{v}}(c+)-R_{u}(c-) Q_{2 n} C_{\bar{\nu}}(c-) \\
& \quad=\rho R_{u}(c+) Q_{2 n} C_{\bar{\nu}}(c+)-R_{u}(c+) C^{*} Q_{2 n}(C+E) C_{\bar{\nu}}(c+) \\
& =-R_{u}(c+) C^{*} Q_{2 n} E C_{\bar{\nu}}(c+) .
\end{aligned}
$$

Let $E \rightarrow 0$, then we get (4.8).

\section{Conclusion}

The dependence of eigenvalues with respect to the data plays an important role in the theory of differential operators. It gives theoretical support for the numerical computation of eigenvalues. Moreover, the properties of monotonicity of eigenvalues with respect to the parameters can be obtained by the derivatives of eigenvalues on the given parameter.

In this article, we obtained the continuity results of eigenvalues and eigenfunctions and presented some new differential expressions of the eigenvalues with respect to the data. Our results in this article generalize the previous results by Kong et al. [24] into a discontinuous version. It can be verified that it turns into the classical case when $\rho=1$.

Acknowledgements

The authors thank the referees for their comments and detailed suggestions. These have significantly improved the presentation of this paper. The work of the authors is supported by the National Nature Science Foundation of China (No. 11561050, No. 11401325). 
Authors' contributions

All authors contributed equally to the writing of this paper. The authors read and approved the final manuscript.

\section{Publisher's Note}

Springer Nature remains neutral with regard to jurisdictional claims in published maps and institutional affiliations.

Received: 18 May 2017 Accepted: 15 September 2017 Published online: 29 September 2017

\section{References}

1. Likov, AV, Mikhailov, YA: The Theory of Heat and Mass Transfer. Qosenerqoizdat, Russian (1963)

2. Aydemir, K, Mukhtarov, OS: Variational principles for spectral analysis of one Sturm-Liouville problem with transmission conditions. Adv. Differ. Equ. 2016, 76 (2016)

3. Aydemir, K, Mukhtarov, OS: Qualitative analysis of eigenvalues and eigenfunctions of one boundary value transmission problem. Bound. Value Probl. 2016, 82 (2016)

4. Bairamov, E, Uğurlu, E: The determinants of dissipative Sturm-Liouville operators with transmission conditions. Math. Comput. Model. 53, 805-813 (2011)

5. Buschmann, D, Stolz, G, Weidmann, J: One-dimensional Schrödinger operators with local point interactions. J. Reine Angew. Math. 467, 169-186 (1995)

6. Uğurlu, E, Bairamov, E: Spectral analysis of eigenparameter dependent boundary value transmission problems. J. Math. Anal. Appl. 413, 482-494 (2014)

7. Li, G, Wang, D, Zhu, B: Well-posedness and decay of solutions for a transmission problem with history and delay. Electron. J. Differ. Equ. 2016, 23 (2016)

8. Li, K, Sun, J, Hao, XL, Bao, QL: Spectral analysis for discontinuous non-self-adjoint singular Dirac operators with eigenparameter dependent boundary condition. J. Math. Anal. Appl. 453, 304-316 (2017)

9. Mukhtarov, OS, Yakubov, S: Problems for ordinary differential equations with transmission conditions. Appl. Anal. 81, 1033-1064 (2002)

10. Wang, A, Sun, J, Zettl, A: Two-interval Sturm-Liouville operators in modified Hilbert spaces. J. Math. Anal. Appl. 328, 390-399 (2007)

11. Wang, Y: Inverse problems for discontinuous Sturm-Liouville operators with mixed spectral data. Inverse Probl. Sci. Eng. 23, 1180-1198 (2015)

12. Zhang, $X$ : The self-adjointness. dissipation and spectrum analysis of some classes high order differential operators with discontinuity, 13-28. Ph.D. thesis (2013) (in Chinese)

13. Zhang, M: Regular approximation of singular Sturm-Liouville problems with transmission conditions. Appl. Math. Comput. 247, 511-520 (2014)

14. Zhang, M, Wang, Y: Dependence of eigenvalues of Sturm-Liouville problems with interface conditions. Appl. Math. Comput. 265, 31-39 (2015)

15. Zhang, M, Sun, J, Zettl, A: Eigenvalues of limit-point Sturm-Liouville problems. J. Math. Anal. Appl. 419, 627-642 (2014)

16. Zheng, Z, Zhang, W: Characterization of eigenvalues in spectral gap for singular differential operators. Abstr. Appl. Anal. 2012, Article ID 271657 (2012)

17. Zettl, A: Ajoint and self-adjoint boundary value problems with interface conditions. SIAM J. Appl. Math. 16, 851-859 (1968)

18. Cai, J, Zheng, Z: A singular Sturm-Liouville problem with limit circle endpoints and eigenparameter dependent boundary conditions. Discrete Dyn. Nat. Soc. 2017, Article ID 9673846 (2017)

19. Li, K, Sun, J, Hao, XL: Eigenvalues of regular fourth order Sturm-Liouville problems with transmission conditions. Math. Methods Appl. Sci. 40, 3538-3551 (2017)

20. Bonnet, A, Dkhil, F, Logak, E: Front instability in a condensed phase combustion model. Adv. Nonlinear Anal. 4(3), 153-176 (2015)

21. Kong, Q, Wu, H, Zettl, A: Dependence of the $n$th Sturm-Liouville eigenvalue on the problem. J. Differ. Equ. 156, 328-354 (1999)

22. Zettl, A: Sturm-Liouville Theory. Mathematical Surveys and Monographs, vol. 121. Am. Math. Soc., Providence (2005)

23. Radulescu, V: Finitely many solutions for a class of boundary value problems with superlinear convex nonlinearity. Arch. Math. (Basel) 84, 538-550 (2005)

24. Kong, Q, Wu, H, Zettl, A: Dependence of eigenvalues on the problem. Math. Nachr. 188, 173-201 (1997)

25. Dauge, M, Helffer, B: Eigenvalues variation. I. Neumann problem for Sturm-Liouville operators. J. Differ. Equ. 104, 243-262 (1993)

26. Kong, Q, Zettl, A: Dependence of eigenvalues of Sturm-Liouville problems on the boundary. J. Differ. Equ. 126, 389-407 (1996)

27. Kong, Q, Zettl, A: Eigenvalues of regular Sturm-Liouville problems. J. Differ. Equ. 131, 1-19 (1996)

28. Suo, J, Wang, W: Eigenvalues of a class of regular fourth-order Sturm-Liouville problems. Appl. Math. Comput. 218, 9716-9729 (2012)

29. Bailey, PB, Everitt, WN, Zettl, A: The SLEIGN2 Sturm-Liouville code. ACM Trans. Math. Softw. 21, 143-192 (2001)

30. Greenberg, L, Marletta, M: The code SLEUTH for solving fourth order Sturm-Liouville problems. ACM Trans. Math. Softw. 23, 453-493 (1997)

31. Naimark, MA: Linear Differential Operators. Ungar, New York (1968)

32. Kong, Q, Zettl, A: Linear ordinary differential equations. In: Agarwal, RP (ed.) Inequalities and Applications, WSSIAA, vol. 3, pp. 381-397. World Scientific, Singapore (1994)

33. Dieudonné, J: Foundations of Modern Analysis. Academic Press, New York (1969) 